\title{
Surface electromyography of facial muscles during natural and artificial feeding of infants
}

\author{
Avaliação eletromiográfica com eletrodos de captação de superfície \\ dos músculos masseter, temporal e bucinador de lactentes \\ em situação de aleitamento natural e artificial
}

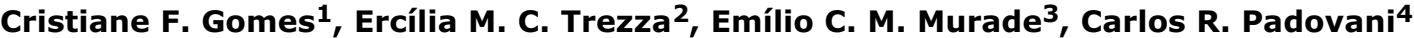

\section{Resumo}

Objetivo: Mensurar e comparar a atividade dos músculos masseter, temporal e bucinador em diferentes métodos de alimentação de lactentes.

Método: Estudo transversal, com participação de 60 lactentes nascidos a termo e sem intercorrências, entre 2 e 3 meses de idade, divididos em três grupos: 1 ) aleitamento materno exclusivo; 2) aleitamento misto com uso de mamadeira; e 3) aleitamento materno exclusivo com uso de copo. Foi realizada eletromiografia com eletrodos de captação de superfície durante a alimentação. O teste estatístico utilizado é o Kruskal-Wallis, complementado com as comparações múltiplas entre pares de grupos, e todas as discussões são realizadas no nível de $5 \%$ de significância.

Resultados: Verificam-se maiores resultados no grupo de aleitamento materno em relação ao grupo de aleitamento por mamadeira, tanto na amplitude quanto na média de contração do músculo masseter. No que se refere ao músculo temporal, há resultados maiores na amplitude do grupo de aleitamento materno e na média de contração do grupo de aleitamento por copo, quando comparados ao grupo de aleitamento por mamadeira. Quanto ao músculo bucinador, observamse resultados maiores no grupo de aleitamento por mamadeira com relação ao aleitamento materno, sendo que tal diferença ocorre apenas na amplitude de contração.

Conclusão: As semelhanças entre a atividade muscular do grupo de aleitamento materno e aleitamento por copo permitem sugerir o uso do copo como método alternativo na alimentação de lactentes, ao contrário do aleitamento por mamadeira, devido à hiperfunção do músculo bucinador, podendo resultar em alterações motoras orais e das funções neurovegetativas.

J Pediatr (Rio J). 2006;82(2):103-9: Eletromiografia, aleitamento materno, músculos faciais, sucção, lactentes.

1. Doutora, Faculdade de Medicina de Botucatu, Universidade Estadual Paulista (UNESP), São Paulo, SP.

2. Doutora. Professora assistente, Faculdade de Medicina de Botucatu, UNESP, São Paulo, SP

3. Doutor, Faculdade de Ciências Médicas da Santa Casa de São Paulo, São Paulo, SP. Docente, Departamento de Ortopedia e Traumatologia, Universidade de Marília (UNIMAR), Marília, SP.

4. Professor titular, Departamento de Bioestatística, Faculdade de Medicina de Botucatu, UNESP, São Paulo, SP

Parte da tese de doutorado defendida em 11 de novembro de 2005 na Faculdade de Medicina de Botucatu, UNESP, São Paulo, SP. Apoio: CAPES

Artigo submetido em 26.09.05, aceito em 14.12.05.

Como citar este artigo: Gomes CF, Trezza EM, Murade EC, Padovani CR. Surface electromyography of facial muscles during natural and artificial feeding of infants. J Pediatr (Rio J). 2006;82:103-9.

\section{Abstract}

Objective: To measure and compare the activity of the masseter, temporalis and buccinator muscles in different infant feeding methods.

Method: Cross-sectional study of 60 full-term infants with no intercurrent diseases, aged between two and three months, classified into the following groups: 1 ) exclusive breastfeeding; 2 ) breastfeeding plus bottle-feeding; and 3 ) exclusive breastfeeding plus cup feeding. Surface electromyography was performed during infant feeding. The Krushal-Wallis test was used, complemented by multiple paired comparisons of the groups. A $5 \%$ significance level was chosen for the tests.

Results: Statistically higher results were verified in the breastfeeding group in relation to the bottle-feeding one, both in the range of movement and the mean contraction of the masseter. With regard to the temporalis muscle, statistically higher results were found in the breastfeeding group comparatively to the bottle-feeding one. As to the buccinator muscle, statistically higher results were observed in the breastfeeding group in relation to the bottle-feeding one, although in this case, the difference concerned only the range of contraction.

Conclusion: The similarities between the muscle activity in the breastfeeding and in the cup-feeding groups suggests that cupfeeding can be used as an alternative infant feeding method, being better than bottle-feeding, due to the hyperactivity of the buccinator muscle, which could result in changes to the structural growth and development of the stomatognathic system functions.

J Pediatr (Rio J). 2006;82(2):103-9: Electromyography, breastfeeding, facial muscles, sucking, infants.

\section{Introdução}

O sistema estomatognático abrange estruturas orais estáticas e dinâmicas, ou seja, as partes duras (arcos dentários, maxila, mandíbula, osso hióide e ossos cranianos) e ativas (músculos, espaços orgânicos, nervos e vasos sangüíneos) que desempenham as funções neurovegetativas (sucção, mastigação, deglutição e respiração), fonação e expressão facial. Tais estruturas estão interligadas de modo que, se houver um distúrbio em alguma delas, todas apresentarão uma desorganização ou desequilíbrio ${ }^{1-3}$.

Para que haja crescimento e desenvolvimento craniofacial, há necessidade de estímulos genéticos e externos, que são oferecidos pela respiração, sucção (amamentação), 
deglutição e mastigação. Os estímulos proporcionados pelos músculos são considerados os maiores responsáveis pelo crescimento, pois realizam tração sobre os ossos, promovendo crescimento ou desgaste, dependendo do local em que a tração ocorre ${ }^{2}$.

No que se refere às conseqüências do aleitamento materno no crescimento das estruturas e desenvolvimento das funções do sistema estomatognático, sabe-se que o crescimento facial harmônico ocorre por meio de movimentos realizados pelo bebê na ordenha, momento em que os maxilares são estimulados a crescer de forma bem direcionada. Além disso, o aleitamento materno proporciona amadurecimento oral, estimulando a tonicidade muscular e o desenvolvimento da articulação temporomandibular, promovendo espaço suficiente para a erupção dentária ${ }^{4-12}$.

A eletromiografia é o estudo da função muscular por meio da análise do sinal elétrico produzido durante a contração muscular. Para a realização do exame, utiliza-se o eletromiógrafo, aparelho capaz de detectar as variáveis elétricas que ocorrem na célula no decorrer da transmissão nervosa e da contração muscular, que são transformadas em sinais elétricos e, após amplificação, são registrados na tela do osciloscópio do aparelho, para serem posteriormente analisados.

A eletromiografia com eletrodos de captação de superfície é utilizada atualmente na pesquisa de diversas patologias: alterações respiratórias, distúrbios do sono, pesquisa de músculos específicos em atletas e animais, na fala de crianças com alterações de oclusão, na avaliação de métodos de alimentação em lactentes, entre outras.

O uso da eletromiografia em pesquisas é mais recente e permitiu determinar a ação da musculatura oral nas diversas formas de alimentação, bem como comparar força e atividade entre elas.

Considerando as especificidades da eletromiografia com eletrodos de captação de superfície na avaliação objetiva da atividade muscular e a escassez de trabalhos que demonstrem as diferenças entre as atividades dos músculos responsáveis pela sucção no aleitamento materno, aleitamento por mamadeira e por copo em lactentes, este estudo tem por objetivo mensurar a atividade muscular dos músculos masseter, temporal e bucinador durante aleitamento materno exclusivo, aleitamento misto com uso de mamadeira e aleitamento materno exclusivo com uso de copo como método alternativo temporário de alimentação.

\section{Métodos}

Para o desenvolvimento deste estudo, utiliza-se o método transversal ou seccional cego, caracterizado por "estudos em que causa e efeito estão presentes no mesmo momento, que é o momento analisado"13.

Participaram da pesquisa 60 lactentes, entre 2 e 3 meses de idade, nascidos a termo e sem nenhum tipo de intercorrência, os quais foram divididos em três grupos: Grupo 1: 20 bebês em aleitamento materno exclusivo; Grupo 2: 20 bebês em aleitamento misto, com oferecimento de complemento por mamadeira; Grupo 3: 20 bebês em aleitamento materno exclusivo que pudessem ser alimentados por copo no momento da realização do exame.

A amostra utilizada foi de conveniência, visto que foram encaminhadas 212 cartas de convite para participação na pesquisa e 64 mães compareceram com seus bebês, sendo que quatro lactentes foram retirados da pesquisa em razão da ocorrência de aleitamento artificial exclusivo (três lactentes) e impossibilidade de conclusão do exame (um lactente). Os critérios de inclusão foram: lactentes entre 60 e 90 dias de vida no momento do exame, em aleitamento materno exclusivo ou misto; nascidos a termo, com peso de nascimento maior que $2.500 \mathrm{~g}$; ausência de intercorrências pré, peri e pós-natais. Foram excluídos da amostra: os gemelares; os que estavam em aleitamento artificial exclusivo e aqueles nos quais foi impossível a realização do exame.

As diferenças detectadas na atividade do músculo masseter em lactentes alimentados ao seio materno e com mamadeira, no estudo de Sakashita et al. ${ }^{14}$, serviram de base para o cálculo do tamanho amostral desta pesquisa. Aceitando-se $\alpha=5 \%$ e $\beta=80 \%$, foi estipulado o número de 18 lactentes por grupo, porém foram analisados 20 lactentes em cada grupo para manter uma margem de segurança.

Realizaram-se 60 exames de eletromiografia no período de 17 de fevereiro a 31 de julho de 2003. Os participantes da pesquisa foram caracterizados quanto à idade (em dias de vida no momento do exame), sexo, idade gestacional de nascimento, tipo de parto, peso de nascimento, perímetro cefálico, comprimento, índice de Apgar no primeiro minuto e uso de chupeta. Além disso, as mães foram caracterizadas quanto à idade, escolaridade e tipo de convênio médico adotado na ocasião do parto.

A pesquisa foi realizada no Hospital Universitário da Universidade de Marília (UNIMAR), mediante autorização da mesma para seu desenvolvimento, com aprovação do comitê de ética em pesquisa da UNESP - Faculdade de Medicina de Botucatu e autorização da Secretaria Municipal de Higiene e Saúde de Marília para a consulta das fichas do Banco de Leite Humano, referentes ao Programa Nascer Cidadão, programa que visa cadastrar e encaminhar os recém-nascidos de risco para as unidades de saúde do município de Marília com objetivo de reduzir a mortalidade infantil.

Inicialmente, a mãe foi orientada quanto aos objetivos da pesquisa e da importância de sua participação e, após seu consentimento verbal, foi solicitada a assinatura do termo de consentimento livre e esclarecido.

Para a realização da pesquisa, foram utilizados: um aparelho de eletromiografia portátil de dois canais da marca Dantec (modelo LBM - Firmware version 4.03, 1993 by Neuro Diagnostics, Inc.) com filtro de $70 \mathrm{~Hz}$, tempo de $5 \mathrm{~ms}$ por divisória e sensitividade de $50 \mu \mathrm{v}$ (microvolts) por divisória; eletrodos de captação de superfície monopolares tipo disco (Eletrodos Maxxigold, marca MF), de ouro, com $9 \mathrm{~mm}$ de diâmetro e com $16 \mathrm{~mm}$ de distância entre o referência e o ativo; creme condutor (Creme Maxxifix, marca MF); fita adesiva para fixação dos eletrodos (Transpore ${ }^{\circledR}$, marca $3 \mathrm{M}$ ); papel termo sensível 
branco para equipamentos de eletromiografia Thermwhite (Alfamedic Ind. Com. e Serviços Ltda.) para a impressão dos resultados.

A atividade eletromiográfica foi graduada e tabulada quantitativamente por um profissional especialista da área de eletromiografia, para posterior análise estatística e comparação entre os diferentes grupos e os músculos estudados.

O profissional especialista realizou uma análise independente, ou seja, sem conhecimento prévio do método de alimentação utilizado no momento da realização do exame, o que tornou a análise livre de interferências do pesquisador.

O teste estatístico utilizado foi a análise de variância não-paramétrica, ou seja, análise para dados com distribuição não-normal (teste de Kruskal-Wallis), complementada com as comparações múltiplas entre pares de grupos ${ }^{15}$.

Foram utilizadas também as medianas, semi-amplitudes interquartílicas, valores inferior e superior, primeiro e terceiro quartis e valores mínimos e máximos das variáveis quantitativas de acordo com os grupos de estudo.

Todas as discussões foram realizadas no nível de $5 \%$ de significância, e os intervalos construídos no nível de $95 \%$ de confiança.

\section{Resultados}

\section{Caracterização dos sujeitos}

A análise estatística demonstrou que não ocorreram diferenças entre os grupos estudados e a variável idade (medianas de 82,50 dias para o grupo de aleitamento materno exclusivo, 83,00 dias para o grupo de aleitamento misto com uso de mamadeira e 84,00 dias para o grupo de aleitamento materno exclusivo com uso de copo), devido ao fato de a variável ser um critério de inclusão, ou seja, apenas lactentes entre 30 e 90 dias participaram da pesquisa (Tabela 1).

\section{Resultados dos exames}

A análise dos músculos foi dividida em: amplitude de contração e média de contração do músculo masseter, amplitude de contração e média de contração do músculo temporal e amplitude de contração e média de contração do músculo bucinador.

\section{Amplitude e média de contração do músculo masseter}

No que se refere à amplitude de contração do músculo masseter, o teste estatístico revela resultado significante na comparação dos grupos segundo o tipo de aleitamento, permitindo afirmar que os grupos de aleitamento por copo (mediana de 3,07 $\mu \mathrm{v}$ ) e aleitamento por mamadeira (mediana de 1,94 $\mu \mathrm{v}$ ) diferem entre si.

Além disso, no que se refere à média de contração do músculo masseter, os grupos de aleitamento materno (mediana de $80 \mu \mathrm{v}$ ) diferem do grupo de aleitamento por mamadeira (mediana de $50 \mu \mathrm{v}$ ) (Tabela 2).

Tabela 1 - Distribuição das freqüências absolutas e porcentagens referentes à caracterização dos sujeitos da pesquisa $(n=60)$

\begin{tabular}{|c|c|c|}
\hline Características & f & $\%$ \\
\hline \multicolumn{3}{|l|}{ Idade do lactente } \\
\hline 60-70 dias & 3 & 5,00 \\
\hline $71-80$ dias & 10 & 16,67 \\
\hline $81-90$ dias & 47 & 78,33 \\
\hline \multicolumn{3}{|l|}{ Sexo do lactente } \\
\hline Feminino & 22 & 36,67 \\
\hline Masculino & 38 & 63,33 \\
\hline \multicolumn{3}{|l|}{ Idade gestacional } \\
\hline 38 semanas & 12 & 20,00 \\
\hline 39 semanas & 18 & 30,00 \\
\hline 40 semanas & 30 & 50,00 \\
\hline \multicolumn{3}{|l|}{ Tipo de parto } \\
\hline Vaginal & 22 & 36,67 \\
\hline Cesárea & 38 & 63,33 \\
\hline \multicolumn{3}{|l|}{ Peso nascimento } \\
\hline $2.500-3.000 \mathrm{~g}$ & 19 & 31,67 \\
\hline $3.001-3.599 \mathrm{~g}$ & 29 & 48,33 \\
\hline $3.600-4.000 \mathrm{~g}$ & 8 & 13,33 \\
\hline$+4.000 \mathrm{~g}$ & 4 & 6,67 \\
\hline \multicolumn{3}{|l|}{ Perímetro cefálico } \\
\hline $33-35,9 \mathrm{~cm}$ & 36 & 60,00 \\
\hline $36-37,9 \mathrm{~cm}$ & 18 & 30,00 \\
\hline Não informado & 6 & 10,00 \\
\hline \multicolumn{3}{|l|}{ Comprimento } \\
\hline $44-47,9 \mathrm{~cm}$ & 15 & 25,00 \\
\hline $48-51,9 \mathrm{~cm}$ & 35 & 58,33 \\
\hline $52-53,9 \mathrm{~cm}$ & 10 & 16,67 \\
\hline \multicolumn{3}{|l|}{ Apgar $1^{\circ}$ minuto } \\
\hline 7 & 4 & 6,67 \\
\hline 8 & 17 & 28,33 \\
\hline 9 & 35 & 58,33 \\
\hline não informado & 4 & 6,67 \\
\hline \multicolumn{3}{|l|}{ Idade materna } \\
\hline-18 anos & 4 & 6,67 \\
\hline $19-25$ anos & 38 & 63,33 \\
\hline 26-30 anos & 8 & 13,33 \\
\hline $31-35$ anos & 8 & 13,33 \\
\hline+35 anos & 2 & 3,33 \\
\hline \multicolumn{3}{|l|}{ Escolaridade materna } \\
\hline $1^{\circ} \mathrm{grau}$ incompleto & 3 & 5,00 \\
\hline $1^{\circ}$ grau completo & 9 & 15,00 \\
\hline $2^{\circ}$ grau completo & 39 & 65,00 \\
\hline Superior incompleto & 2 & 3,33 \\
\hline Superior completo & 7 & 11,67 \\
\hline \multicolumn{3}{|l|}{ Convênio médico } \\
\hline SUS & 23 & 38,3 \\
\hline Particular & 14 & 23,3 \\
\hline Convênio & 16 & 26,7 \\
\hline Não informado & 7 & 11,7 \\
\hline \multicolumn{3}{|l|}{ Uso de chupeta } \\
\hline Mamadeira & 10 & 16,67 \\
\hline Amamentação & 0 & - \\
\hline Copo & 0 & - \\
\hline
\end{tabular}


Tabela 2 - Mediana, semi-amplitude interquartílica, valores mínimo e máximo, limites inferior e superior, primeiro e terceiro quartis das variáveis amplitude de contração e média de contração do músculo masseter $(n=20)$

\begin{tabular}{|c|c|c|c|c|c|}
\hline \multirow{2}{*}{$\begin{array}{l}\text { Variável } \\
(n=20)\end{array}$} & \multirow{2}{*}{$\begin{array}{c}\text { Medida } \\
\text { descritiva }\end{array}$} & \multicolumn{3}{|c|}{ Grupos } & \multirow{2}{*}{$\begin{array}{c}\text { Resultado do } \\
\text { teste estatístico } \\
\text { p }\end{array}$} \\
\hline & & $\begin{array}{l}\text { Aleitamento } \\
\text { exclusivo }\end{array}$ & Mamadeira & Copo & \\
\hline \multirow{8}{*}{$\begin{array}{l}\text { Amplitude de contração: } \\
\text { masseter }\end{array}$} & LI & $*$ & $*$ & $*$ & $6,61(p<0,05)$ \\
\hline & Valor mínimo & 0,84 & 0,77 & 1,70 & \\
\hline & Q1 & 1,62 & 1,40 & 2,12 & \\
\hline & Mediana & $2,38 a b$ & $1,94 \mathrm{a}$ & $3,07 b^{+}$ & \\
\hline & Q3 & 3,14 & 2,80 & 4,31 & \\
\hline & Valor máximo & 4,48 & 5,27 & 9,10 & \\
\hline & LS & 5,42 & 4,90 & 7,42 & \\
\hline & Sem. interq. & 0,76 & 0,70 & 1,10 & \\
\hline \multirow{8}{*}{$\begin{array}{l}\text { Média de contração: } \\
\text { masseter }\end{array}$} & LI & $*$ & 1,25 & $*$ & $7,35(p<0,05)$ \\
\hline & Valor mínimo & 31,88 & 19,17 & 42,50 & \\
\hline & Q1 & 57,60 & 42,50 & 53,03 & \\
\hline & Mediana & $80,00 \mathrm{~b}$ & $50,00 \mathrm{a}$ & $76,59 a b$ & \\
\hline & Q3 & 99,90 & 70,0 & 107,82 & \\
\hline & Valor máximo & 298,33 & 131,67 & 227,50 & \\
\hline & LS & 163,35 & 111,50 & 202,80 & \\
\hline & Sem. interq. & 21,15 & 13,75 & 27,40 & \\
\hline
\end{tabular}

$\mathrm{LI}=$ limite inferior; $\mathrm{LS}$ = limite superior; Sem. interq. = semi-amplitude interquatílica.

* Valores negativos: valores impossíveis de serem obtidos.

$†$ Duas medianas seguidas de pelo menos uma mesma letra não diferem no nível $5 \%$ de significância.

\section{Amplitude e média de contração do músculo temporal}

No que se refere à amplitude de contração do músculo temporal, observam-se diferenças na comparação dos grupos, permitindo afirmar que os grupos de aleitamento materno (mediana de 4,02 $\mu \mathrm{v}$ ) e de aleitamento por copo (mediana de $3,84 \mu \mathrm{v}$ ) diferem do grupo de aleitamento por mamadeira (mediana de 1,81 $\mu \mathrm{v}$ ).

Com relação à média de contração do músculo temporal, os grupos de aleitamento materno (mediana de 111,25 $\mu \mathrm{v}$ ) e aleitamento por copo (mediana de 96,04 $\mu \mathrm{v}$ ) diferem do grupo de aleitamento por mamadeira (mediana de 48,03 $\mu \mathrm{v}$ ) (Tabela 3).

\section{Amplitude e média de contração do músculo bucinador}

Observando-se a Tabela 4, no que se refere à amplitude de contração do músculo bucinador, há diferenças na comparação entre os grupos, permitindo afirmar que o grupo de aleitamento por mamadeira (mediana de 1,70 $\mu \mathrm{v}$ ) difere do grupo de aleitamento materno (mediana de $1,20 \mu \mathrm{v})$.

No que se refere à média de contração do músculo bucinador, não são observadas diferenças nas medianas entre os grupos segundo o tipo de aleitamento após a realização da análise estatística (Tabela 4).

\section{Discussão}

Observou-se que, na comparação entre os grupos segundo o tipo de aleitamento, no que se refere ao aleitamento materno, diversas pesquisas comprovam que há maior participação do músculo masseter, visto que este atua na ordenha por meio da movimentação da mandíbula, mais especificamente na elevação e protrusão mandibular, sendo caracterizado como músculo de força e resistente à tração ${ }^{5-11}$.

De acordo com Palmer ${ }^{16}$, Madeira ${ }^{17}$ e Almeida et al. ${ }^{18}$, os movimentos mandibulares favorecem o adequado crescimento e posicionamento mandibular para a erupção dentária, por isso a importância da participação do músculo masseter na alimentação de lactentes, proporcionado apenas no aleitamento materno e aleitamento por copo, já que no aleitamento por mamadeira, além de uma diminuição na atividade do masseter, há aumento da atividade dos bucinadores, diminuição dos movimentos mandibulares, retração de língua com possibilidade de hipofunção e hiperfunção, de acordo com o tipo de sucção realizado ${ }^{19}$.

Os resultados descritos anteriormente são semelhantes aos encontrados na literatura, sendo que, no estudo de Sakashita et al. ${ }^{14}$, os resultados eletromiográficos revelam a ocorrência de maior atividade do músculo masseter no aleitamento materno do que no aleitamento por mamadei- 
ra, concluindo que os bebês em aleitamento por mamadeira podem apresentar alterações no desenvolvimento da função mastigatória, gerando possíveis distúrbios de mastigação e deglutição.

Da mesma forma, a pesquisa desenvolvida por Tamura et al. ${ }^{20}$ demonstra que, no aleitamento por mamadeira, o músculo masseter apresenta atividade significativamente diminuída quando comparada ao aleitamento materno.

Apesar de a maioria das pesquisas corroborarem os dados desta pesquisa, Sakashita et al. ${ }^{14}$ concluíram em seu estudo, que compara a sucção de bebês em aleitamento materno, aleitamento por mamadeira com bico comum e

Tabela 3 - Mediana, semi-amplitude interquartílica, valores mínimo e máximo, limites inferior e superior,
primeiro e terceiro quartis das variáveis amplitude de contração e média de contração do músculo
temporal $(\mathrm{n}=20)$

$\mathrm{LI}=$ limite inferior; $\mathrm{LS}$ = limite superior; Sem. interq. = semi-amplitude interquatílica

*Valores negativos: valores impossíveis de serem obtidos.

Tabela 4 - Mediana, semi-amplitude interquartílica, valores mínimo e máximo, limites inferior e superior, primeiro e terceiro quartis das variáveis amplitude de contração e média de contração do músculo bucinador $(n=20)$

\begin{tabular}{|c|c|c|c|c|c|}
\hline \multirow{2}{*}{$\begin{array}{l}\text { Variável } \\
(n=20)\end{array}$} & \multirow{2}{*}{$\begin{array}{c}\text { Medida } \\
\text { descritiva }\end{array}$} & \multicolumn{3}{|c|}{ Grupos } & \multirow{2}{*}{$\begin{array}{c}\text { Resultado do } \\
\text { teste estatístico } \\
\mathbf{p}\end{array}$} \\
\hline & & $\begin{array}{l}\text { Aleitamento } \\
\text { exclusivo }\end{array}$ & Mamadeira & Copo & \\
\hline \multirow{8}{*}{$\begin{array}{l}\text { Amplitude de contração: } \\
\text { bucinador }\end{array}$} & LI & $*$ & $*$ & $*$ & \multirow[t]{8}{*}{$6,89(p<0,05)$} \\
\hline & Valor mínimo & 0,57 & 1,23 & 0,50 & \\
\hline & $\mathrm{Q} 1$ & 0,82 & 1,47 & 0,92 & \\
\hline & Mediana & $1,20 \mathrm{a}$ & $1,70 \mathrm{~b}$ & $1,36 a b$ & \\
\hline & Q3 & 1,85 & 2,76 & 2,25 & \\
\hline & Valor máximo & 10,35 & 3,30 & 2,86 & \\
\hline & LS & 3,41 & 4,71 & 4,23 & \\
\hline & Sem. interq. & 0,52 & 0,65 & 0,66 & \\
\hline \multirow{8}{*}{$\begin{array}{l}\text { Média de contração: } \\
\text { bucinador }\end{array}$} & LI & $*$ & $*$ & $*$ & \multirow[t]{8}{*}{$5,13(p>0,05)$} \\
\hline & Valor mínimo & 20,00 & 30,83 & 12,50 & \\
\hline & Q1 & 30,42 & 36,88 & 23,12 & \\
\hline & Mediana & 42,09 a & 47,82 a & 33,92 a & \\
\hline & Q3 & 65,54 & 75,37 & 56,25 & \\
\hline & Valor máximo & 258,75 & 92,00 & 71,50 & \\
\hline & LS & 118,22 & 133,12 & 105,93 & \\
\hline & Sem. interq. & 17,56 & 19,25 & 16,56 & \\
\hline
\end{tabular}

$\mathrm{LI}=$ limite inferior; $\mathrm{LS}$ = limite superior; Sem. interq. = semi-amplitude interquatílica.

* Valores negativos: valores impossíveis de serem obtidos. 
aleitamento por mamadeira com um novo tipo de bico artificial, que a atividade do músculo masseter é maior no grupo de bebês em aleitamento por mamadeira com o novo tipo de bico artificial do que no grupo de aleitamento materno.

No que se refere ao aleitamento por copo, Kuehl 21 afirma que os movimentos da língua e mandíbula realizados no aleitamento por copo são semelhantes aos movimentos necessários ao aleitamento materno bem-sucedido, sugerindo que o aleitamento por copo pode ser uma oportunidade para que o bebê desenvolva musculatura necessária a esses movimentos.

Os dados obtidos permitem afirmar também que, na impossibilidade da ocorrência do aleitamento materno em alguns horários, o aleitamento por copo pode ser indicado, pois apresenta valores de amplitude e média de contração do músculo masseter maiores do que no aleitamento por mamadeira, permanecendo intermediário entre este e o grupo de aleitamento materno.

No que se refere ao músculo temporal, poucos estudos enfocam sua atividade, possivelmente por ser mais caracterizado como músculo de movimento do que de força, sendo responsável, assim como o músculo masseter, pela elevação da mandíbula pelo engajamento de suas fibras com as do masseter e pterigóideo medial16-18. O músculo temporal possui tanto a função de elevação mandibular por suas fibras verticais quanto de retrusão por suas fibras oblíquas e horizontais ${ }^{19,22}$.

Apesar de não haver informações de estudos de eletromiografia durante o aleitamento por copo, a presente pesquisa permite inferir que, para os momentos de necessidade de alimentação através de um método alternativo, o aleitamento por copo seria mais indicado do que o aleitamento por mamadeira, pois, além de impedir a chamada confusão de bicos, proporciona a participação dos músculos masseter e temporal de forma semelhante à participação dos mesmos músculos no aleitamento materno.

Ao contrário, no aleitamento por mamadeira, a participação dos músculos masseter e temporal é menor quando comparada aos demais métodos de alimentação, semelhantemente ao que Carvalho ${ }^{19}$ descreve ao afirmar que, no aleitamento materno, os músculos masseteres, temporais, pterigóideos, língua, lábios, mentalis e bucinadores apresentam-se em normofunção, e no aleitamento por mamadeira com bico convencional, os músculos masseteres, temporais, pterigóideos, língua e lábios apresentam-se em hipofunção, e o mentalis e bucinadores em hiperfunção.

Ahlgren ${ }^{23}$, que investiga atividade eletromiográfica dos músculos periorais, entre eles o bucinador, durante sucção nutritiva e não-nutritiva de crianças com má-oclusão e oclusão normal, constata que, durante a sucção e deglutição, o músculo bucinador apresenta atividade maior em crianças com má-oclusão do que em crianças com oclusão normal, destacando a relação entre a atividade do bucinador com a ocorrência de alterações de oclusão, em concordância com as informações de Carvalho ${ }^{19}$.
Conclui-se que, na comparação entre os grupos de aleitamento, há concordância com a literatura acerca da atividade dos músculos estudados no aleitamento materno e aleitamento por mamadeira, embora não tenham sido encontrados artigos científicos que corroborem os resultados da pesquisa com aleitamento por copo.

Os dados obtidos permitem concluir que, tanto no aleitamento materno quanto no aleitamento por copo, os mesmos músculos atuam de maneira semelhante (sem diferenças estatisticamente significantes), demonstrando uma maior participação dos masseteres e temporais e reduzida participação dos bucinadores.

O aleitamento materno continua sendo, portanto, o método de alimentação mais adequado para lactentes, e o uso do copo é recomendável como substituto do aleitamento materno nos momentos de impossibilidade deste, pois a musculatura ativa em ambos os métodos é a mesma, com a vantagem de não provocar a "confusão de bicos".

Percebe-se, com relação ao método utilizado para a coleta e análise dos dados, que esse se mostrou adequado para responder aos questionamentos iniciais. No entanto, alguns vieses podem ter interferido nos resultados da pesquisa, como, por exemplo, o uso de chupeta por parte de metade dos lactentes do grupo de aleitamento misto com uso de mamadeira, fato que pode ter promovido mudanças no padrão de sucção dos lactentes, ainda que os mesmos já utilizassem mamadeira.

Apesar disso, novas pesquisas deverão ser desenvolvidas com o objetivo de verificar a movimentação da língua e outras estruturas orais em ambos os métodos de alimentação, observando se há diferenças no aleitamento por mamadeira e copo.

Outros estudos ainda deverão ser realizados com prematuros, bebês com síndromes ou más-formações de cabeça e pescoço, com a finalidade de verificar a possibilidade de oferecimento do copo como método de alimentação e sua eficácia em populações específicas de lactentes.

Ainda com relação ao uso do copo, deve-se levar em consideração que não deve ser utilizado como método único de alimentação de lactentes, pois nele não ocorre sucção ou ordenha; ao contrário, o bebê apenas sorve o leite. O que se destaca, então, é a importância do uso do copo como método alternativo e temporário ao aleitamento materno, que deve ser retomado assim que as condições permitam.

\section{Referências}

1. Bianchini EMG. Crescimento e desenvolvimento craniofacial. In: Bianchini EMG. A cefalometria nas alterações miofuncionais orais: diagnóstico e tratamento fonoaudiológico. $2^{a}$ ed. São Paulo: Pró-Fono; 1994. p. 5-15.

2. Tanigute CC. Desenvolvimento das funções estomatognáticas. In: Marchesan IQ. Fundamentos em fonoaudiologia: aspectos clínicos da motricidade oral. Rio de Janeiro: Guanabara-Koogan; 1998. p. 1-6.

3. Carvalho GD. Amamentação e o sistema estomatognático. In: Carvalho MR, Tamez RN. Amamentação: bases científicas para a prática profissional. Rio de Janeiro: Guanabara-Koogan; 2002. p. 37-49. 
4. Vardes $V$, Sanchez AP, Labbok M. Manejo clínico da lactação: assistência à nutriz e ao lactente. Rio de Janeiro: Revinter; 1996. p. 1-25.

5. Righard L, Alade MO. Sucking technique and its effect on success of breastfeeding. Birth. 1992;19:185-9.

6. Fernandes FBU. Pensando no bebê: benefícios, técnicas e dificuldades do aleitamento materno [monografia]. Rio de Janeiro: 2000. Centro de Especialização em Fonoaudiologia Clínica; 2000.

7. Matida MY. Amamentação: uma visão fonoaudiológica [monografia]. Londrina: Centro de Especialização em Fonoaudiologia Clínica; 2000.

8. Medeiros EB, Rodrigues MJ. Importância da amamentação natural para o desenvolvimento do sistema estomatognático do bebê. Rev Cons Reg Odontol Pernambuco. 2001;4:79-83.

9. Gava-Simioni LR, Jacinto SR, Gaviao MBD, Puppin Rontani RM. Amamentação e odontologia. J Bras Odontopediatr Odontol Bebê. 2001;4:125-31.

10. Jacinto-Goncalves SR, Gaviao MBD, Berzin F, Oliveira AS, Semeguini TA. Electromyographic activity of perioral muscles in breastfed and non-breastfed children. J Clin Pediatr Dent. 2004; 29:57-62.

11. Woolridge MW. The 'anatomy' of infant sucking. Midwiferi. $1986 ; 2: 164-71$.

12. Naylor AJ, Danner S, Lang S. Development of oral motor function. In: Naylor AJ, Morrow AL. Developmental readiness of normal full term infants to progress from exclusive breastfeeding to the introduction of complementary foods: reviews of the relevant literature concerning infant gastrointestinal, immunologic, oral motor and maternal reproductive and lactational development. San Diego: Wellstart International; 2001. p. 21-6.

13. Campana AO. Pesquisa clínica - estudos observacionais. In: Campana AO. Introdução à investigação científica. Botucatu: UNESP; 1995. p. 87-100.

14. Sakashita R, Kamegai T, Inoue N. Masseter muscle activity in bottle feeding with the chewing type bottle teat: evidence from electromyographs. Early Hum Dev. 1996;45:83-92.
15. Norman GR, Streiner DL. Bioestatistics: the bare essentials. St. Louis: Mosby-Year Book; 1994.

16. Palmer JM. Oral cavity. In: Palmer JM. Anatomy for speech and hearing. 14th ed. Baltimore: Williams \& Wilkins; 1993. p. 53-90.

17. Madeira MC. Músculos da face. In: Madeira MC. Anatomia da face: bases anátomo-funcionais para a prática odontológica. $3 a$ ed. São Paulo: Sarvier; 2001. p. 67-95.

18. Almeida EOC, Melli R, Moraes IF. Orientação fonoaudiológica e psicológica às nutrizes: experiência em contexto hospitalar. In: Tasca SMT, Almeida EOC, Servilha EAM. Recém-nascido em alojamento conjunto: visão multiprofissional. Carapicuíba: PróFono; 2002. p. 35-49.

19. Carvalho GD. O sistema estomatognático e suas funções. In: Carvalho GD. S.O.S. respirador bucal: uma visão funcional e clínica da amamentação. São Paulo: Lovise; 2003. p. 27-56.

20. Tamura Y, Horikawa Y, Yoshida S. Co-ordination of tongue movements and peri-oral muscle activities during nutritive sucking. Dev Med Child Neurol. 1996;38:503-10.

21. Kuehl J. Cup feeding the newborn: what you should know. J Perinat Neonatal Nurs. 1997;11:56-60.

22. Sanches MTC. Manejo clínico das disfunções orais na amamentação. J Pediatr (Rio J). 2004;80:S155-62.

23. Ahlgren J. EMG studies of lip and cheek activity in sucking habits. Swed Dent J. 1995;19:95-101.

Correspondência:

Cristiane F. Gomes

Rua José Pereira da Costa, 89, casa 7, Residencial Paulista II

CEP 87005-220 - Maringá, PR

Tel.: (44) 3025.7095

Fax: (44) 3028.4888

E-mail: cfg.fono@flash.tv.br 